\title{
5G WIRELESS NETWORKS DEPLOYMENT WITH EMERGING TECHNOLOGIES AND RESEARCH CHALLENGES
}

\author{
${ }^{1}$ Dr Rajamohan Parthasarathy*, ${ }^{2}$ Ms Preethy Ayyappan, ${ }^{3} \mathrm{Mr}$ Seow Soon Loong \\ ${ }^{1}$ School of Information Technology, SEGi University \\ ${ }^{2}$ Faculty of Engineering and Built in Environment, SEGi University \\ ${ }^{3}$ School of Information Technology, SEGi University \\ * prajamohan@segi.edu.my
}

\begin{abstract}
Over the last couple of decades the world has witnessed gradual, yet steady evolution of mobile wireless communications towards second, third and fourth generation wireless networks. Introduction of digital modulations, effective frequency reuse, penetration of packetbased Internet and rapid advancement in physical layer technologies. Currently all IP based 4G Long Term Evolution (LTE/4G) networks have become a part of everyday life. As a result, a set of new, user-oriented mobile multimedia applications, like mobile video conferencing, streaming video, e-healthcare and online gaming are coming up. These new applications are not only satisfying users' requirements, but also opening up new business horizons for wireless operators to increase their revenue. Fifth generation mobile communications $(5 \mathrm{G})$ are expected to accommodate rapidly increasing mobile traffic aiming at the realization of a "Hyper Connected World" in which all people and surrounding things are connected and information is exchanged between them, and to play the role of a basis of the Internet of everything. The 5G wireless network is expected to become a "Heterogeneous Network" where new wireless access technologies incompatible with $4 \mathrm{G}$ and the wireless access technologies for unlicensed band are incorporated with the enhanced technology of 4G (e.g. IMT-advanced). This paper introduces the vision and emerging technology trends of $5 \mathrm{G}$, shows key directions of the research challenges and development of $5 \mathrm{G}$ in the future.
\end{abstract}

Keywords: Heterogeneous Network (HetNets), Fifth Generation Mobile Communications (5G), Long Term Evolution (LTE/4G), massive Machine-Type Communications (mMTC), Internet of Things (IoT) 


\section{Introduction}

The mobile phone has evolved rapidly over the past decade from a monochrome device with a minuscule screen and little processing power to one with high resolution, palm sized screen and processing power rivalling a laptop. This transformation, coupled with an expanding cache of bandwidth hungry applications have triggered demands for higher data rates. Mobile data traffic has been forecasted to grow more than 24-fold between 2010 and 2015, and more than 500-fold between 2010 and 2020 [1]. This has in turn propelled the uptake of 4G contracts and driven operators worldwide to deploy $4 \mathrm{G}$ networks. As the dust around $4 \mathrm{G}$ begins to settle, attention is now slowly turning towards future $5 \mathrm{G}$ technologies.

While there is no industry consensus on what $5 \mathrm{G}$ will ultimately be, apart from the usual higher data rate and energy efficiency, there are some emerging signs of things to come. For example, in the new IEEE 802.11 High Efficiency Wireless (HEW) study group, there is a pronounced increase in the presence of cellular operators, something not previously seen. This indicates growing interests to amalgamate different technologies to support future connectivity and data rates. Therefore, we believe that $5 \mathrm{G}$ will consist of multiple interconnected communication standards, ranging from wireless metropolitan area networks down to wireless personal networks, providing the required throughput and connectivity.

To cope with the high traffic volume demands spurred by the proliferation and penetration of $4 \mathrm{G}$ services, significant improvements to wireless access techniques have been made. Notable examples are massive multiple-input multiple-output (MIMO), coordinated multipoint (CoMP) transmission and reception, and D2D (device-to-device) incorporated in $3 \mathrm{G}$ and $4 \mathrm{G}$ specifications. Furthermore, to boost network coverage and capacity, mobile operators have also been increasingly deploying heterogeneous networks (HetNets), consisting of micro-, pico-, and small/femtocells. However, despite these advances in core wireless technologies, Third Generation Partnership Project (3GPP) mobile networks are still challenged to handle the ever increasing mobile traffic, particularly due to the use of specific-purpose networking equipment that can neither dynamically scale with mobile traffic nor easily be upgraded with new functions.[2]

Researchers have been designing new architectures for elastically composing and operating a virtual end-to-end network platform on demand on top of fragmented physical infrastructures provided by federated clouds. Software-defined networking (SDN) techniques have been seen as promising enablers for this vision of carrier cloud, which will likely play a crucial role in the design of $5 \mathrm{G}$ wireless networks. [2] 


\subsection{Defining 5G}

$5 \mathrm{G}$ is the term used to describe the next (and fifth) generation of wireless networks, beyond current $4 \mathrm{G}$ LTE networks. Although a final standard for $5 \mathrm{G}$ is yet to be formally designed, [3] $5 \mathrm{G}$ networks are expected to build on, and smoothly integrate with, the legacy of previous generations of wireless network. In the first instance, $5 \mathrm{G}$ represents an evolution of existing radio access technologies. From $2 \mathrm{G}$ to $4 \mathrm{G}$, each radio access technology generation over the last 25 years has focused on improving the speed and efficiency of wireless networks to enhance mobile services. Evolution of cell data rates under different radio access technologies.

Source: DotEcon and Axon

- $2 \mathrm{G}$ Voice $64 \mathrm{Kbps}$

- $\quad 3 \mathrm{G}$ Voice and Data $2 \mathrm{Mbps}$

- 4G Data 1 Gbps

- $\quad 5 \mathrm{G}$ Data and more... Up to 20 Gbps

Each transition has been driven by the greater capabilities of each generation over its predecessor. 3G enabled mobile internet and data connectivity at a much more advanced level than 2G. [4] 4G was able to serve the massive increase in mobile data traffic, something $3 \mathrm{G}$ was not capable of.

With more people using mobile broadband as services and applications expand, more devices of various types connecting to mobile networks and continued growth in data, further enhancements will eventually be required. Operators are looking ahead to identify ways their networks can be readied to meet future capacity and performance requirements.

The deployment of $5 \mathrm{G}$ networks is seen as important in fulfilling expected mobile data traffic growth. However, $5 \mathrm{G}$ differs from previous generations in important ways. Improvements to existing mobile broadband services to provide enhanced mobile broadband (eMBB) is just one use-case for $5 \mathrm{G}$. In the longer run, $5 \mathrm{G}$ is expected provide tailored connectivity to meet the particular demands of different user groups, including particular industries (so-called 'verticals'). Verticals may need data connectivity both to use internally for their own operations and to embed within the products and services they supply to their end customers.

5G will create opportunities for network operators to tap new sources of revenue by developing infrastructure that can deliver a range of innovative services for enterprises, including IoT (Internet of Things) applications and deep integration of connectivity (e.g. transport telematics). For the first time, mobile networks will be designed to address the varying 
needs of different industries and the rise of connected devices (so-called massive machine-type communications - mMTC).

As more devices and objects need to be securely, automatically and remotely connected and monitored -allowing systems, machines and infrastructures to run with end-to-end machine-tomachine communication - a large number of sectors may become increasingly reliant on wireless network solutions. $5 \mathrm{G}$ is projected to co-exist with the $4 \mathrm{G}$ networks, but to deliver improved connectivity through high speed, reliable and secure communications to approximately one-third of the global population by 2025. [5]

5G is able to bring enhanced capabilities including lower latency, high resource efficiency, decreased energy consumption, and enhanced security. This can drive access to a broad range of applications and services, as well as opening opportunities for new business models within the telecommunications sector and key verticals.

The software and IT industry in Europe stands to evolve because the $5 \mathrm{G}$ network services will rely on software and virtualisation, creating an expanded ecosystem. This may create options to develop new partnerships and synergies across industries. $5 \mathrm{G}$ will allow for a shift from networks designed and built from the outset for specific performance characteristics to agile networks that can be programmatically assembled and configured for specific use cases. In effect, a common network infrastructure can carry multiple 'virtual' networks with differing performance characteristics aimed at different types of users, with this being readily reconfigurable without needing further physical investment.

The current 4G/LTE networks cannot provide instantaneous cloud services, tactile Internet, enhanced vehicle-to-everything (eV2X), Internet of things (IoT) and communication with drones and robots while guaranteeing quality of experience to mobile users. Furthermore, LTE networks can provide high-quality video experience to only a limited number of mobile users simultaneously. In spite of such advanced $4 \mathrm{G}$ wireless network technologies, it is hard to provide mobile services that require high speed, rapid response, high reliability and energy efficiency. Hence, these features have become essential requirements for future services in the $5 \mathrm{G}$ era.

By introducing new contents, such as 360-degree videos and holograms, and new service concepts, such as smart transportation and machine-type communications, future $5 \mathrm{G}$ services can be developed in several directions such as unlimited data transmission, a massive number of active connections and new types of mobile devices, especially sensors, powered by sustainable energy sources. The scope of $5 \mathrm{G}$ services is not limited to personal communications, but extends to the areas of societies including mobile phones, wearable devices, sensors, actuators, vehicles, 
robots, and so on. Therefore, $5 \mathrm{G}$ networks can be regarded as the key infrastructure that innovates societies, as well as ICT industries.

Research on $5 \mathrm{G}$ services and their technical requirements has been performed by the International Telecommunication Union-Radio communication Sector (ITU-R), the 3rd Generation Partnership Project (3GPP) and the Next Generation Mobile Networks (NGMN) Alliance. In the ITU-R Working Party (WP) 5D, 5G is defined by the name of International Mobile Telecommunications-2020 (IMT-2020), and various 5G services are presented in a vision document [6]. The proposed usage scenarios are grouped into three categories: enhanced mobile broadband (eMBB), massive machine-type communications (mMTC) and ultra-reliable and low-latency communications (URLLC). Peak data rate, area traffic capacity, network energy efficiency, connection density, latency, mobility, spectrum efficiency and user-experienced data rate are selected as key performance indicators (KPIs), which can be regarded as technical requirements. As a result of this analysis, $5 \mathrm{G}$ services are categorized according to the following five features: immersiveness, intelligence, omnipresence, autonomy and publicness. Each category includes typical $5 \mathrm{G}$ services as follows.

- Immersive 5G services: Virtual Reality/Augmented Reality (VR/AR), massive contents streaming

- Intelligent 5G services: User-centric computing, crowded area services

- Omnipresent 5G services: Internet of things

- Autonomous 5G services: smart transportation, drones, robots

- Public 5G services: disaster monitoring, private security/public safety, emergency services

\subsection{Analysis on the Megatrend of Mobile Services}

In this section, various changes to our daily life that are brought by $5 \mathrm{G}$ services are described by investigating five megatrends of mobile services as follows:

- Explosion of mobile data traffic

- Rapid increase in connected devices

- $\quad$ Everything on the cloud

- Hyper-realistic media for convergence services

- Knowledge as a service enabled by big data analysis

As mobile multimedia contents streaming and social networks have become more popular, the demand for mobile traffic has continuously increased. In addition to this, new types of 
multimedia services such as augmented reality, virtual reality and holograms, which all require huge traffic volumes, have emerged. According to [7], the amount of mobile data traffic in 2020 (30.6 exabytes per month, $1 \mathrm{~EB}=1,000,000 \mathrm{~TB})$ will be 8.3-times higher than in $2015(3.7$ exabytes per month).

In addition to mobile traffic volume, the number of mobile devices has increased exponentially by introducing new types of devices such as wearable devices, sensors, vehicles, drones and robots. In particular, this trend has accelerated because many machines with sensors and/or actuators are connected to communication networks for IoT services [8]. Globally, it is expected that the market of mobile handsets and other IoT devices, which are connected to the Internet, may increase from USD7.9 billion in 2015 to USD11.6 billion in 2020 [7,9]. The increase of active connections requires another direction of network evolution.

To access mobile networks with high computing power through simple portable devices, mobile cloud computing has emerged as one of the most essential technologies [10]. It is expected that the portion of mobile cloud traffic with respect to total mobile traffic will increase from $35 \%$ in 2013 to $70 \%$ by 2020 [11]. Furthermore, most mobile services in the 5G era are expected to be integrated with the mobile cloud computing system.

Unlike current 2D multimedia, hyper-realistic media services like AR, VR and multi-point view are enabled by the development of mobile networks and media technologies. Interactive services based on 3D holograms are also expected to become more popular. These hyperrealistic media services are tightly coupled with other sectors of the industry such as robots, drones, vehicles, health-care, personal assistance and security [12]. It is expected that big data analytics will create new opportunities in various areas because the value of data can be extracted through data gathering, processing, predictive analysis, inference, etc. The potential of big data analysis, especially in the area of knowledge services and artificial intelligence, is maximized by employing cloud computing technology, e.g., machine learning, deep learning, inference and recognition of IBM Watson [13][14].

\section{Heterogeneous Networks}

\subsection{Small Cells}

As the demand for higher data rates increases, one of the solutions available to operators is to reduce the size of the cell. By reducing the size of the cell, area spectral efficiency is increased through higher frequency reuse, while transmit power can be reduced such that the power lost through propagation will be lower. Additionally, coverage can be improved by deploying small cells indoors where reception may not be good and offloading traffic from macro cells when 
required. This solution has only been made possible in recent years with the advancement in hardware miniaturization and the corresponding reduction in cost. Additionally, changes to the functional architecture of the access network allowed data and control signals to tunnel through the Internet, enabling small cells to be deployed anywhere with Internet connectivity. Small cells can have different flavors, with low powered femtocells typically used in residential and enterprise deployments, and the higher powered picocells used for wider outdoor coverage or filling in macro cell coverage holes. The concurrent operation of different classes of base stations, macro-, pico-, and femto- base stations, is known as heterogeneous networks (or HetNets). This is used to provide a flexible coverage area and improve spectral efficiency. Overlaying different classes of base stations can also potentially provide a solution for the growing data traffic, especially when the transport of data is optimized to take advantage of the characteristics of heterogeneous networks. 3GPP has identified various scenarios and requirements for the enhancement of small cells in [16].

\subsection{New Carrier Type}

One of the key concepts underpinning the operation of enhanced small cells is the separation of the control plane and the user plane. The control plane provides the connectivity and mobility while the user plane provides the data transport. In such a scenario, the user equipment (UE) will maintain connection with two different base stations, a macro and a small cell, simultaneously. The macro cell will maintain connectivity and mobility (control plane) using lower frequency bands, while the small cell provides high throughput data transport using higher frequency bands [15]. An alternative version is the splitting of uplink and downlink across different classes of base stations.

The motivation behind this is that in the current 3GPP standard, cell specific reference signals are always transmitted regardless of whether there are data to transmit or not, and transmitters cannot be switched off even when there is no data to transmit. However, with the definition of a new carrier type [17], where cell specific control signals, such as reference and synchronization signals, are removed, this is no longer the case. The macro cells will now provide the reference signals and information blocks, while the small cells, using the new carrier, can deliver data at higher spectrum efficiency, throughput, and energy savings. Additionally, they can now be switched off when there is no data to transmit. This can also provide additional benefits such as lower interference [18]. Such a scheme is expected to improve cell edge user throughput by up to 70 percent and reduce macro node energy consumption by 20 percent at low loads [18]. 


\subsection{Multiple Radio Access Technologies}

Although the 3GPP define heterogeneous networks as the concurrent operation of different classes of base stations, we believe that heterogeneous networks in $5 \mathrm{G}$ will be a mixture of different radio access technologies as well. This will include future Wireless Local Area Network (WLAN) technologies which can offer seamless handovers to and from the cellular infrastructure, and device to device communications. This will lighten the burden on cellular networks and shift load away from the treasured licensed bands. At the same time, it can also concurrently provide higher throughput to users. This can already be implemented in part using the 3GPP Access Network Discovery and Selection Function (ANDSF) [19]. However, in situations where there is a high concentration of user terminals, offloading of data to WLANs may result in poor throughput, as WLANs are not well equipped to handle a large number of users. This problem is recognized by the IEEE 802.11 Working Group, which has initiated a study group on High Efficiency WLANs (HEW) to tackle situations where there is a high density of access points and/or a high density of user terminals.

\subsection{Device to Device Communications}

Another approach to solving the highly dense network problem will be through Device to Device (D2D) communications, where each terminal is able to communicate directly with other terminals in order to either share their radio access connection, or to exchange information. Coupled with power control, D2D communications can reduce interference, especially in nonlicensed frequency bands. In 4G cellular communications, there are no provisions made for devices to communicate directly with nearby devices. All communications will have to be routed through the base station, and the gateway. This is extremely inefficient, especially when the devices are close by.

In scenarios such as machine to machine (M2M) communications, where the number of devices involved can potentially be very large, it would be more sensible if devices can communicate directly with each other when necessary. In unlicensed spectrum, devices can already communicate with each other outside of the cellular standard using technologies such as Bluetooth or Wireless LAN in ad hoc mode. However, these connections are susceptible to interference. On the other hand, using licensed spectrum will guarantee a certain level of quality of service if the connection is managed properly. These D2D communications will almost certainly require the base station to facilitate the connections to avoid intra-cell interference. The process to standardize this approach is already set in motion in 3GPP [20] 


\subsection{Challenges of Heterogeneous Networks}

\subsubsection{Inter-cell Interference}

One of the biggest problems for HetNets is inter-cell interference. This is especially problematic with unplanned deployment of small cells, where the operators have little or no control of the location of the small cell. Additionally, the concurrent operation of small cells and traditional macro cells will produce irregular shaped cell sizes, and hence inter-tier interference, which will require advanced power control and resource allocation to avoid inter-cell interference.

\subsubsection{Distributed Interference Coordination}

In deployment of access points where there are little or no coordination, such as between WLANs, distributed interference avoidance will be required. This will be increasingly crucial as more devices access unlicensed spectrum to complement their throughput.

\subsubsection{Efficient Medium Access Control}

This is particularly relevant for dense deployment of access points and user terminals where the medium access is distributed, such as that of WLANs. In such situations, the user throughput is low, latency is high, and hotspots will not be able to complement cellular technology to provide a high throughput. Existing medium access control will need to be redesigned for such an environment to optimize the channel usage.

\subsubsection{Device Discovery and Link Setup}

In non-network assisted device discovery in D2D communications, there could be issues when there is a large number of devices around. Additionally, setting up and maintain links with more than one party can prove to be difficult, especially when operating in the same frequency

\section{Software Defined Cellular Networks}

In parallel with the development of software defined radio in wireless communications, Software Defined Networking (SDN) has gathered momentum in the networking industry in the past few years. The concept of SDN originates from Stanford University's Open Flow system [24], which enables abstraction of low level networking functionality into virtual services. In this way, the network control plane can be decoupled from the network data plane, which significantly simplifies network management and facilitates the easy introduction of new 
services or configuration changes into the network. Currently in both academia and industry, a clear definition of SDN is still lacking. Nevertheless, according to a standardization body of SDN, Open Networking Foundation (ONF), the SDN architecture has the following features [25]:

\subsection{Directly programmable}

The network control plane is logically centralized and decoupled from the data plane. Network intelligence resides in software-based SDN controllers that maintain a global view of the network.

\subsection{Open:}

SDN simplifies network design and operation via open standards-based and vendor-neutral APIs (northbound and southbound).

\subsection{Agile:}

Network operators can dynamically configure, manage, and optimize network resources and adjust traffic flows to meet changing needs quickly via dynamic and automated SDN programs.

Recently, there are also growing interests in both academia and industry to apply SDN to mobile networks. The main motivation behind this is that SDN may help cellular operators simplify their network management and enable new services to support the exponential traffic growth envisaged for $5 \mathrm{G}$ networks. The authors of [26] argue that with open APIs and virtualization, SDN can separate the network service from the underlying physical infrastructure, thereby moving towards a more open wireless ecosystem and facilitating fast innovation. Similar to the programmable switches in wired SDN networks, programmable base stations and packet gateways are envisioned in cellular SDN architectures with extensions such as network virtualization on subscriber attributes and flexible adaptation of air interfaces [27]. Therefore, we believe that wireless or cellular SDN could be a possibility in future wireless networks.

Future 5G applications may have diverse characteristics and quality of service (QoS) requirements. For instance, M2M traffic has very different latency, throughput, and priority features compared to Human to Human $(\mathrm{H} 2 \mathrm{H})$ traffic. The same can be said for real-time video traffic and usual web browsing data traffic. The flexibility offered by SDN can enable finegrained resource control (e.g. based on subscriber attributes) to enhance user Quality of Experience (QoE) while in the meantime maximizing network utilization [27]. 
At the moment, the wireless industry has yet to reach a consensus on a unified view of future 5G network architecture. Some favor a more distributed network architecture with selforganizing capability, while others have advocated more centralized cloud-based access networks. The development of cellular SDN is somewhat orthogonal to this ongoing evolution as it provides an open, flexible, and programmable middleware solution that can be used in different network architectures. Two important issues are scalability (to support a large number of small cells and a huge number of devices) and robustness (to provide a reliable abstraction without negatively impacting the flexibility).

\subsection{Challenges of Software Defined Networking}

Wireless SDN is still at its infancy. There are a number of outstanding issues to be resolved before it can realize its full potential [28]. Firstly, further development is needed to apply SDN concepts at network infrastructure level, e.g. introducing SDN into carrier networks. For example, there is currently no consensus yet on how the programmable switch can be achieved in the best way (in terms of performance and flexibility trade-off). Secondly, global standardization is still on-going and a unified cellular programmable interface for implementing SDN infrastructures has yet to emerge. In particular, the standard development of a reliable Network Operation System (NetworkOS) that provides unified access to computing, storage and network resources is crucial for SDN implementation in a multi-vendor environment [28]. Finally, security in SDN is an open problem.

\section{Massive MIMO and 3D MIMO}

Another technology which is being considered is the use of a large array of antenna elements, several orders more than the number in use today, to provide diversity and compensate for path loss [21]. Otherwise known as Massive Multiple-Input/Multiple-Output (MIMO), it also allows for high resolution beamforming and is especially useful at higher frequencies where antenna elements can be miniaturized.

\subsection{Massive MIMO}

Massive MIMO can purportedly increase the capacity by several orders and simultaneously improve the radiated energy-efficiency [22]. In addition, it provides large number of degrees of freedom, which can be exploited using beamforming if the channel state information is available. Another advantage of Massive MIMO is its energy efficiency, and each antenna element is expected to use extremely low power [22]. 
However, there are several research challenges which need to be solved before Massive MIMO can be incorporated into future wireless systems. Beamforming will require a large amount of channel state information, and this will be problematic especially for the downlink. Consequently, Massive MIMO may be impractical for FDD systems, but can be used in TDD systems due to the channel reciprocity. Alternatively, limited feedback can be used. Additionally, Massive MIMO suffers from pilot contamination from other cells if the transmit power is high, and will suffer from thermal noise otherwise [22].

\subsection{D MIMO}

3D MIMO which allows for 3D beamforming. This is sometimes considered as a special type of large scale MIMO which is only concerned with using the antenna elements for beamforming. While normal beamforming methods form beams in two dimensions, 3D MIMO allows beam control in both horizontal and vertical directions. This additional control allows for further sectorization within a cell. As with Massive MIMO, 3D MIMO requires new channel models. Currently, 3GPP has started a work item on modelling 3D channels [23]. 3D MIMO will also require additional modifications to the feedback mechanism.

\subsection{Challenges of Massive/3D MIMO}

\subsubsection{Channel Estimation/Feedback}

Currently, only time division duplexing scenarios are considered for massive MIMO due to the prohibitive cost of channel estimation and feedback. Even for time division duplexing to work, channel calibration for Massive MIMO can prove to be a feat. New methods of channel estimation and feedback schemes will need to be proposed for massive MIMO to achieve mainstream status.

\subsubsection{Fast Processing Algorithms}

To deal with the massive amount of data from the RF chains, extremely fast algorithms to process these data will be required.

\subsubsection{Pilot Contamination}

Massive MIMO suffers from pilot contamination from other cells. Work around for this will be required for Massive MIMO to deliver its promised performance. 


\section{Machine to Machine Communications}

As the enabling technologies described above continue to develop apace, fueling the growth of service coverage and capacity, new use cases and applications are being identified, their emergence demanding yet more of our global networks. Many of these new business areas involve autonomous communication between devices, whether these devices are components in a smart energy network, intelligent home appliances or vehicles and infrastructure in an integrated transportation system. Indeed, we are already seeing examples of these "machine to machine" (M2M) devices: consider the latest generation of in-car satellite navigation ("sat-nav") devices with their integral cellular modems, downloading traffic information updates invisibly in the background. These new applications have the potential to cause a step change in the size of the telecommunications market.

There are several challenges specific to $\mathrm{M} 2 \mathrm{M}$ communications, not least of which are the autonomous operation and often restrictive power, size and complexity requirements. The typical M2M traffic is also quite distinctive: having spent the past decades optimizing our networks, from the highest level servers to the lowest level PHY channel codes, to support the characteristic traffic flows linked to speech, browsing and messaging. The distribution and nature of these M2M traffic flows do not sit readily within the current network architectures, so extensions and modifications are required.

The technology extensions developed and deployed to support these M2M applications have been appearing in a relatively ad hoc and piecemeal fashion, in specific standards bodies and/or organizations with particular technical or regional remits. Clearly, this vertical approach, while getting solutions out to market quickly, is not ideal in the long-term. There are areas of commonality in M2M solutions where consistent, standardized and open horizontal approaches will help develop the economies of scale and interoperability that will lead to a truly global M2M market.

It was to this end that the "oneM2M" Partnership Project (PP) was formed during 2012, to develop global, access-technology agnostic Service Layer specifications for M2M, in the same mold as 3GPP. This international body was formed by Standards Development Organisations (SDOs) from across the world: ETSI from Europe, ATIS and TIA from the USA, TTC and ARIB from Japan, CCSA from China and TTA from South Korea. Each of these SDOs already had interests in (and, in many cases, solutions for) different aspects of, and variations on, M2M systems: for example, the ETSI Technical Committee M2M had already produced an entire tranche ("Release 1") of technical requirements and specifications. These existing standards 
range from architectural descriptions to interface definitions, such as service layer interactions with common cellular access systems such as those developed by 3GPP and 3GPP2.

The architecture is based around Common Service Functions (from Device Management to Session Management) residing within Common Service Entities (CSE), with interfaces between the CSE and the applications above and the underlying network services below clearly defined. The current feeling is that both service- and resource-orientated architectures on the key CSE interfaces should be supported.

The system is designed adopting the REST philosophy (a "RESTful" system). That is, the system is stateless, with uniquely addressable entities. Furthermore, the system must have welldefined interfaces between client and server, and between layers, to allow independent development and evolution of components. This RESTful approach is a key enabling technology for not only the "Internet of Things" but also the longer-term "web of things", which we believe will be an application within 5G systems.

Communication flows based around request/response interactions are also defined, and protocols for the different interfaces are being identified and scoped. The key, top-level documents have already been finalized and agreed, and are now under change control processes. Work continues within the different Working Groups to finalize and agree the remainder. The group is anticipated to deliver a first release in the middle of 2014.

\subsection{Challenges of $\mathrm{M} 2 \mathrm{M}$}

Specific challenges vary according to the exact M2M application, but there are two themes that recur in many, if not all, M2M applications:

\subsubsection{Massive access:}

Compared to conventional human to human traffic in cellular networks, a huge number of $\mathrm{M} 2 \mathrm{M}$ devices in a cell can pose serious system challenges in terms of radio access network (RAN) congestion and overload. Currently a number of proposals have been proposed in 3GPP to address the RAN overload issue, e.g. back-off adjustment, access class barring, and M2M prioritization. However, each of these methods has its strengths and weaknesses and none of them is widely acknowledged as the best solution.

\subsubsection{Security and privacy:}

Security has been widely discussed in various standardization bodies. For instance, in ETSI M2M [29], M2M security focuses on several attributes of a user and their communications, 
including authenticity, authority, integrity, and confidentiality. To enable wide deployment of M2M services and especially enhance consumer acceptance, M2M privacy is of paramount importance. Different M2M applications and sectors (e.g. e-health and smart metering) may have different privacy requirements which have to be taken into account right from the beginning of system design.

\section{Other Technologies}

Apart from the above technologies and applications, the following technologies can also potentially impact $5 \mathrm{G}$.

\subsection{Millimeter Wave}

An obvious way of increasing the throughput will be through bandwidth expansion. However, the available bandwidth below $6 \mathrm{GHz}$ is limited, and re-farming analogue TV spectrum will not sufficiently meet the burgeoning demand. Already, there are efforts to look beyond $6 \mathrm{GHz}$ and also at the millimetre wave frequencies to evaluate their feasibility for use in future networks. However, the characteristics of higher frequencies are not well studied, and measurement campaigns and channel modelling for different scenarios and environments will be required before transmission technologies can be designed for them. We believe that millimetre wave frequencies holds the most promise, and there are already ongoing efforts to make this a possibility. In [29], millimetre wave frequencies of $28 \mathrm{GHz}$ and $38 \mathrm{GHz}$ are extensively studied to understand their propagation characteristics in different environments, paving the way for their use in future wireless systems.

\subsection{Shared Spectrum}

Although cognitive radio was often touted as a solution to the problem of frequency spectrum shortage, it is seldom adopted as there are always concerns about the impact on the primary user or license holder of the spectrum. An alternative solution proposed which can potentially solve this dilemma is Authorized Spectrum Access (ASA) also known as Licensed Spectrum Access (LSA) [31]. The concept of LSA is to allow authorized users to access licensed spectrum based on certain conditions set by the licensee of the spectrum. This would allow under-utilized spectrum to be more effectively used and also solve the problem of quality of service for the primary user. 


\subsection{Big data}

Like in many other market sectors and industries, big data will also bring about lots of challenges and opportunities in $5 \mathrm{G}$ wireless. First of all, cellular networks have to provide efficient infrastructure support for this data deluge. For example, the future M2M or Internet of Things (IoT) applications will generate a vast amount of data. As discussed previously, this proves to be a major technical challenge for RANs. Secondly, new network architectures may emerge from the necessity of running big data applications. There is close synergy between cloud computing, software defined networking, and Network Function Virtualization (NFV). A convergence of these technologies can be envisaged to form highly robust and reliable $5 \mathrm{G}$ platforms for big data. Thirdly, making informed decisions and extracting intelligence from big data is an extremely important and yet non-trivial task. For example, cellular operators can make use of various customer network access data to reduce churn rate and seek new revenue opportunities. The smart grid, as another example, can be seen as a huge sensor network, with immense amounts of grid sensor data from various sensors, meters, appliances and electrical vehicles. Data mining and machine learning techniques are essential for efficient and optimized operation of the grid.

\subsection{Indoor Positioning}

While indoor positioning itself does not improve throughput or coverage, it has large implications on various applications and the quality of communications. Accurate positioning of user terminals can provide the network with additional information that can help in resource allocation and quality of service improvement. It can also enable a plethora of applications, including position based handover, resource allocation, and location based services.

Currently, 3GPP LTE has several positioning methods, including Cell ID (CID) and Enhanced Cell ID (ECID), as well as Assisted Global Navigational Satellite Systems (A-GNSS). It is also able to position using the Observed Time Difference of Arrival (OTDOA) method. All these are enabled through the Enhanced Serving Mobile Location Centre (E-SMLC) using the LTE Positioning Protocol (LPP) [32]. Accuracy improvements to the currently available methods will certainly open opportunities for more location based applications.

\section{Conclusions}

In this paper, we have provided an overview of some emerging technologies which may make up future $5 \mathrm{G}$ wireless networks. We have also described some research problems which these technologies present. While there is currently no clear consensus among academics and 
industrials on what will define $5 \mathrm{G}$ wireless networks, we believe that future $5 \mathrm{G}$ wireless networks will be a combination of different enabling technologies, and the biggest challenge will be to make them all work together.

\section{Acknowledgement}

The authors would like to thank SEGi University Management, SEGi Journal of Engineering \& Technological Advances (JETA) Editorial Board, Research Innovation Management Centre (RIMC) SEGi University and School of Information Technology SEGi University.

\section{References}

[1] T. Nakamura, S. Nagata, A. Benjebbour, Y. Kishiyama, H. Tang, X. Shen, N. Yang, and N. Li, "Trends in Small Cell Enhancements in LTE Advanced," IEEE Communications Magazine, vol. 51, no. 2, pp. 98-105, Feb 2013.

[2] Victor C. M. Leung, Tarik Taleb, Min Chen, Homasmagedanz, Li-Chun Wang, Rahim Tafazolli, Unveiling 5g Wireless Networks: Emerging Research Advances, Prospects, And Challenges, Guest Editorial, IEEE Network, pp 3-5, November/December 2014.

[3] Although, 3GPP Release 15 has defined the 5G system architecture. See 3GPP Release 15: Source: www.3gpp.org

[4] The emergence of (limited) data connectivity and mobile internet predated " $3 G$ " in the form of General Packet Radio Service (GPRS) (Release 97).

[5] GSMA Intelligence, Global Mobile Trends, 2017.

[6] Recommendation ITU-R M.2083-0. IMT Vision-Framework and Overall Objectives of the Future Development of IMT for 2020 and Beyond. Available online: http://www.itu.int/rec/R-REC-M.2083- 0201509-I/en.

[7] Cisco. VNI Global Mobile Data Traffic Forecast 2015-2020. Available online:

http://www.cisco.com/c/en/ us/solutions/service-provider/visual-networking-index-vni/index.html 
[8] Gubbi, J.; Buyya, R.; Marusic, S.; Palaniswami, M. Internet of Things (IoT): A vision, architectural elements, and future directions. Future Gener. Comput. Syst. 2013, 29, 1645-1660.

[9] Cisco. Internet of Things. Available online:

http://www.cisco.com/c/dam/en\$_\$us/about/ac79/docs/innov/IoT\$_\$IBSG\$_0411FINAL.pdf

[10] Forbes. 6 Big Internet Trends to Watch for in 2012. Available online: http://www.forbes.com/sites/roberthof/2011/12/13/6-big-internet-trends-to-watch-for2012/\$\#\$63b4b066427c

[11] GSMA. The Mobile Economy; GSMA. Available online:

http://www.gsma.com/mobileeconomy/global/2016, Sustainability 2017, 9, 184821 of 22

[12] Ministry of Science, ICT and Future Planning. Science, ICT R\&D Mid and Long-Term Strategies (2013-2017); Ministry of Science, ICT and Future Planning: Seoul, Korea, 2013.

[13] National IT Industry Promotion Agency (NIPA). Forecast on ICT and Convergence Technology Through Analysis on the Future Technology; National IT Industry Promotion Agency: Jincheon, Korea, 2013.

[14] Intel IT Center. Big Data in the Cloud: Converging Technologies. Available online: http://www.intel.com/content/dam/www/public/us/en/documents/product-briefs/big-datacloudtechnologies-brief.pdf

[15] T. Nakamura, S. Nagata, A. Benjebbour, Y. Kishiyama, H. Tang, X. Shen, N. Yang, and N. Li, "Trends in Small Cell Enhancements in LTE Advanced," IEEE Communications Magazine, vol. 51, no. 2, pp. 98105, Feb 2013.

[16] 3rd Generation Partnership Project, "Scenarios and Requirements for Small Cell Enhancements for EUTRA and E-UTRAN", 3GPP TR36.932 V12.0.0, Dec 2012.

[17] 3rd Generation Partnership Project, “New Carrier Type for LTE,” 3GPP RP 122028, Sep 2012.

[18] C. Hoymann, D. Larsson, H. Koorapaty and J.-F. Cheng, “A Lean Carrier for LTE,” Communications Magazine, IEEE, vol. 51, no. 2, pp. 74-80, 2013. 
[19] 3rd Generation Partnership Project, “Access Network Discovery and Selection Function (ANDSF) Management Object (MO),” 3GPP TS 24.312 V12.1.0, Jun 2013.

[20] 3rd Generation Partnership Project, "Feasibility Study for Proximity Services (ProSe)," 3GPP TR 22.803 V12.2.0, Jun 2013.

[21] T.L. Marzetta, "Noncooperative Cellular Wireless with Unlimited Number of Base Station Antennas," IEEE Trans. Wireless Comm., vol. 9, no. 11, pp. 3590-3600, Nov 2010.

[22] E.G. Larsson, F. Tufvesson, O. Edfors, T.L. Marzetta, "Massive MIMO for Next Generation Wireless Systems," IEEE Communications Magazine, Submitted, 2013.

[23] 3rd Generation Partnership Project, "Study on 3D-channel model for Elevation Beamforming and FDMIMO studies for LTE,” 3GPP RP 122034, Dec 2012.

[24] N. McKeown et al., OpenFlow: enabling innovation in campus networks, ACM CCR, April 2008.

[25] ONF, https://www.opennetworking.org/sdnresources/sdn-definition.

[26] K. Yap et al., Blueprint for Introducing Innovation into Wireless Mobile Networks, ACM VISA, 2010.

[27] L. Li, Z. Mao, and J. Rexford, CellSDN: SoftwareDefined Cellular Networks, Technical report, Bell Labs, 2012.

[28] ADVA et al., Horizon 2020 Advanced 5G Network Infrastructure for Future Internet PPP Industry Proposal (Draft Version 2.1), 2013.

[29] ETSI TC M2M, TR 102 764, E-health Architecture: Analysis of User Service Models, Technologies \& Applications supporting eHealth, 2009.

[30] T.S. Rappaport et al, "Millimeter Wave Mobile Communications for 5G Cellular: It Will Work!," IEEE Access, vol. 1, no. 1, pp. 335-349, May 2013.

[31] Radio Spectrum Policy Group 2011, "Report on Collective Use of Spectrum (CUS) and other spectrum sharing approaches," Technical Report, RSPG11-392, Nov 2011. 
[32] 3rd Generation Partnership Project, "LTE Positioning Protocol (LPP)," 3GPP TS 36.355 V11.3.0, Jul 2013 